\title{
Intelligent Adaptive Information Systems for Educational Purposes
}

\author{
Georgij Yalamov ${ }^{1, a}$, Tamara Shikhnabieva ${ }^{1, b *}$ \\ 1 Institute of Education Management of the Russian Academy of Education, 105062, 16 \\ Zhukovskogo str., Moscow, Russia \\ ${ }^{a}$ geo@portalsga.ru, ${ }^{b}$ shetoma@mail.ru \\ ${ }^{*}$ Corresponding author
}

\begin{abstract}
Keywords: adaptive information systems for educational purposes, didactic completeness of information systems, approaches to intellectualization, pedagogical and technological requirements, principles of didactic systems, intellectualization of educational systems
\end{abstract}

\begin{abstract}
The current state of adaptive information systems for educational purposes (AISEP) on the subject of their didactic completeness, their intellectualization approaches. An approach focused on the inclusion of didactic rules in the AISEP knowledge base has been proposed. The pedagogical and technological requirements for the AISEP capabilities are formulated taking into account the principles of didactic systems.
\end{abstract}

\section{Introduction}

Modern scientific interpretation of adaptive learning involves the personalization of the learning process based on the creation of electronic courses that take into account individual characteristics of students, including the level of basic knowledge, perception, psychological characteristics, as well as individual tasks and learning objectives.

If we talk about modern adaptive e-learning, then this area is currently actively developing in the field of education, based on the knowledge engineering methods used to create educational information systems taking into account the behavior and state of knowledge of a person in the learning process.

\section{Methodology}

The models of adaptive e-learning are implemented on the basis of several modern approaches and methods: (i) the method of expert assessments; (ii) multi-agent approach; (iii) programmed training based on an application package (a traditional approach); (iv) application of fuzzy logic methods based on the theory of fuzzy sets; (v) the use of output technology based on neural network algorithms; (vi) intellectual planning; (vii) ontological engineering; (viii) combining different approaches to create integrated learning systems. The article reviewed and highlighted the most promising of the above approaches.

\section{Research}

In the modern adaptive e-learning, the method of expert assessments, based on the use of expert information systems, allows one to realize the opportunity not to lay out a preliminary sequence of learning steps, since it is built by the information system itself in the course of its operation [1]. This allows one to create for each student a primary individual training plan based on his competence-oriented model, which is based on the analysis of answers to questions from special tests, some personal characteristics of students. The expert tutoring systems (hereinafter, ETS) are capable of performing structural and parametric adaptations, which make it possible to bring the structure and parameters of the student's model to his real "portrait", but the didactic possibilities presented at the same time are very limited. The individual learning plan is adjusted by the ETS based on the results of mapping the current model of a particular learner, based on the experience of his learning, to a reference course model with subsequent comparison with fragments of the applied ontology of the course or discipline.

As a tool for building expert systems, AT-TECHNOLOGY [2] is of interest, which is aimed at the development and use of ETS in the educational process for an automated support of a number of academic disciplines. In particular, the accumulated technological and methodological experience of the automated management of a significant number of individual models of students in these disciplines and relevant 
training models allow, based on the results of its analysis, to use and develop modern innovations in solving intellectual education problems using integrated ETS, including web-integrated ETS. On the basis of the complex facilities, it was possible to implement a specific set of functional tasks characteristic of an intellectual learning technology, including the following: "individual planning of a specific learning methodology", "intelligent analysis of learning tasks solutions", and "intellectual decision support".

In addition, the technology of automated construction of integrated ETS is being developed, using simultaneously the approaches of both knowledge engineering and ontological engineering, intelligent planning and traditional programming [2].

The multi-agent approach makes it possible to implement the ability to adapt all levels (structural and parametric, management objects, learning objectives), which makes it possible to manage the object (student) at all stages of the learning process. The approach is based on the construction of the system as a set of programs (agents). Each agent has its own semantic description of its field of activity (its own structure and knowledge), representing a particular ETS with a traditional structure. In addition to the memory of its activities, the agent also has its own resources to achieve its own goals, has the ability to interact with other agents and resolve conflicts with the goals of other agents to achieve a common goal. This allows one to freely choose those objectives of the learning process that are currently pursued by the control subsystem, and in accordance with these goals, choose a standard (provided by the relevant agent). Compliance with the standard is achieved by the current model of the student. Thus, for each specific task of learning, a certain group of agents is drawn up, which indicates a change in the structure and goals of the crucial subsystem depending on the task.

In the aspect of our research, the cloud platform IACPaaS (Intelligent Application, Control and Platform as a Service) is of interest, which is designed to provide support for the development, management, and remote use of application and instrumental multi-agent cloud services and their components for various subject areas [2]. On the basis of this service, it was possible to create a virtually implemented computerbased training simulator for medical students in order to train their professional knowledge and skills in the process of teaching one of section in medicine [3]. The problem-independent toolkit has also been developed, which allows creating not only training simulators, but also training courses, virtual laboratories, and animated videos. However, this toolkit does not contain the components necessary to create training systems based on the model of the learner and his learning experience.

AISEP can be divided into two subsystems by its functional feature:

1) Data storage, processing, and display subsystem (easily algorithmizable functions);

2) An intellectual subsystem (intellectual functions, i.e. decision-making functions).

These two subsystems interact with each other remaining independent, which allows one to edit the knowledge base and influence the system operation as a whole (changing critical algorithms, data involved in the decision-making process). The universal structure of the intellectual subsystem is invariant to its content, and can be used in various automated information systems without any changes.

Thus, the intellectualization of information systems provides their properties, that is, AISEP is a priori an intellectual system.

The core of intellectual systems for educational purposes (hereinafter, ISE) is fundamentally a knowledge base that supports a constantly evolving domain model. This knowledge base should adequately reflect current knowledge of the subject area. The presence of such subject areas and tasks of automation of the educational process determines the optimal use of this principle when creating ISE. But the intellectualization of the architecture of educational systems, which ensures their adaptive properties, is in most cases hampered by the need to algorithmize data structures of subject areas, the complexity of the structures themselves.

Illustrative in this respect is an example of solving the problems of creating educational Intellectual Systems in the framework of the IDEA project (creating educational expert systems in a number of subject areas). It was aimed at creating automaton models of a teacher and a student [4] interacting through the space of educational material, formalized to the form of marked informational trees or loaded graphs that have a general appearance. Successful examples of learning systems were built in the field of learning 
foreign languages based on ETS. However, these projects could not be developed to its full completion due to the need to create a large set of decision rules, which caused large expenditures of resources. Despite this, research related to expert information systems continues today.

Nevertheless, the use of specialized instrumental high-tech e-learning tools in information systems creates prerequisites, but does not guarantee the high didactic quality of electronic educational resources and the educational process. Moreover, in the process of automated learning, a number of contradictions have arisen that violate the main trends of modern education - integration and differentiation. It should be noted that these contradictions are manifested in traditional education and are associated with a tendency to simplify a number of teaching materials, both in secondary and higher education, caused by a decrease in the level of training of applicants and high workload of the teaching staff of higher educational institutions, a formal approach to quality management education and the struggle for quantitative indicators. Due to the destruction of a number of scientific schools, the break in continuity of education and Russian science, the decline in the quality of dissertations in teaching practice over the years, there has been a decrease in the requirements for training and retraining of teachers and teaching staff.

As an analysis of the experience of using AISEP in educational institutions shows [5; 6; 7 and others], there is no purposeful use of high-tech e-learning tools in managing the educational process in accordance with the required principles of didactic systems [8]. Methods of forming models of students and knowledge bases of subject areas in the considered ISE do not provide for the division of educational content into didactic units of predefined types. Individual learning strategies do not take into account the optimal dosage of knowledge and exercises by the teacher, depending on the student's abilities, the speed of remembering and forgetting knowledge, the stability and duration of his active state. All of this limits the ability to automatically check an e-learning course for didactic completeness and prevents transferring similar didactic units to another course, constructing an individual training plan adequate to the model of the student.

In this regard, an introduction to the ISE learning management subsystem of an adaptive block of intellectual support, the block diagram of which is presented in Figure 1, is relevant.

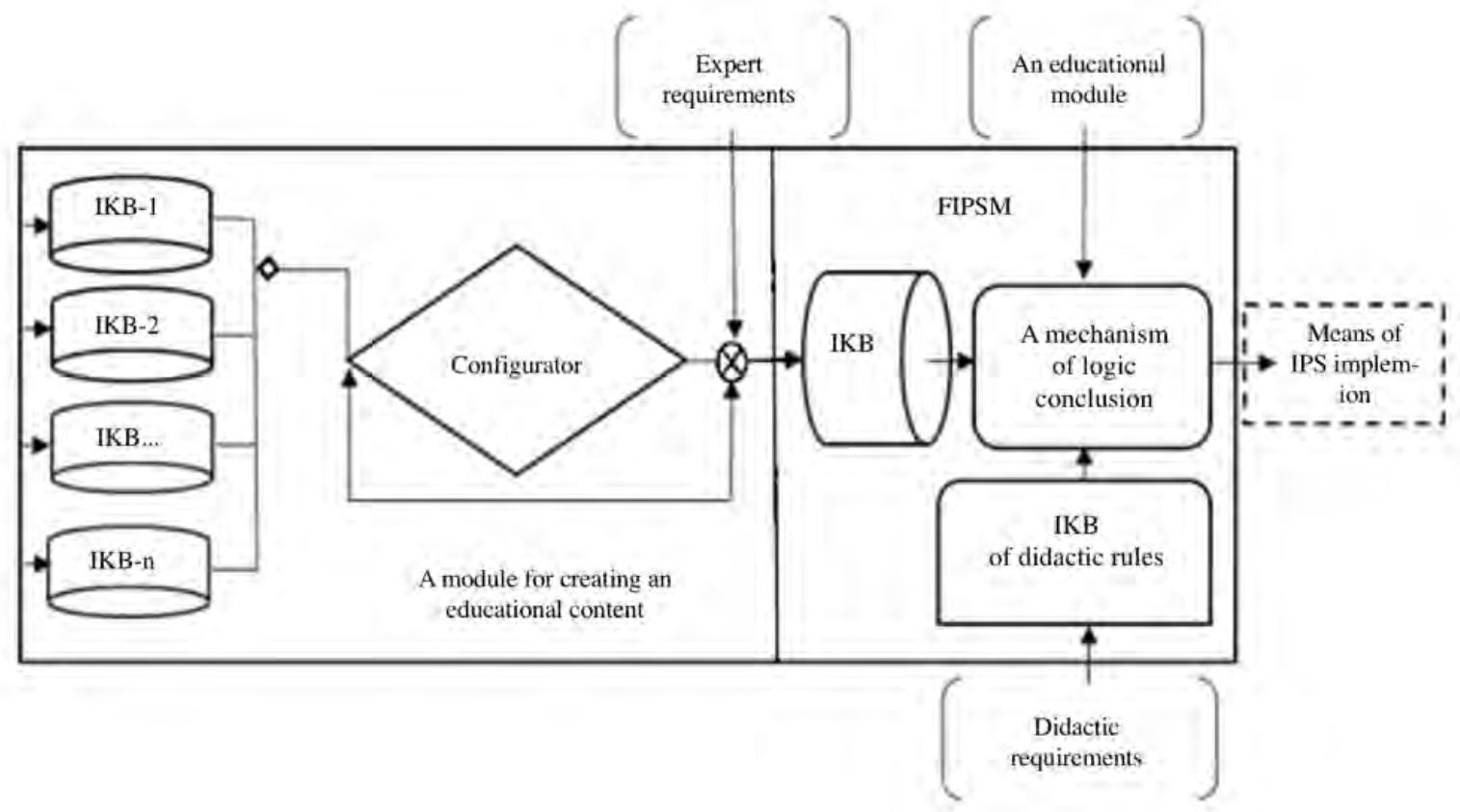

Fig. 1. Block diagram of the adaptive block of intelligent support for the learning management subsystem.

IPS - individual learning path, FIPSM - a forming IPS module, IKB - integrated knowledge base, IKB-n - knowledge bases of various subject areas, LM - learning model.

The main purpose of the functioning of this unit is to build an individual learning path. The peculiarity is that the scheme provides a module for the formation of educational content that configures the knowledge bases of different subject areas, and the inference engine is associated with the knowledge base of didactic 
rules in the form of formalized didactic requirements of an expert (a knowledge engineer), according to which the educational content can be divided into didactic units and taking into account some individual cognitive abilities of the student.

The following approach to the implementation of this block is proposed. A special mechanism for the logical inference of fragments and facts of educational content is used (local verification and assessment of the quality of the educational process based on a comparison of the selected scenario of educational material with the course ontology). Algorithms of inference and learning are formalized in the form of a fairly large set of curves that allow a natural interpretation of the following type: "single error", "progress". The algorithm of sampling and logical inference of fragments of educational content is implemented by the information resource decomposition. The educational content is structured in the form of a set of trees with cross-references. This approach ensures a hierarchical structure of the teaching material and the formation of various kinds of links that create primary, secondary, and other didactic structures of educational material, reflecting the interrelationships of various educational goals, tasks of competences, and control actions.

Depending on the type of a student model and the chosen learning scenario (deductive, inductive, or hybrid), it is proposed to use three learning vectors ("slow", "normal", and "fast").

This approach allows one to simulate the process of real learning, taking into account such didactic features as the mutual integration of verification processes for models of a teacher, a student and a training course, the optimality of a strategy for dosing knowledge and tasks by a teacher, cognitive abilities and the level of basic knowledge of a learner, speed of forgetting and memorizing knowledge and the duration of its active state.

Select the main didactic requirements for ISE:

1) Providing a learner with the most suitable and individually planned sequence of information blocks and learning tasks. The possibility of active and passive construction of such a sequence, depending on the current model of the student.

2) Implementation of an intelligent decision analyzer, providing analysis of the student's final answers to educational tasks. The goal of predictive decision analysis is to determine whether the solution proposed by the student is correct or incorrect, to find out what is wrong or incomplete in the answer; and, perhaps, the determination of missing or incorrect knowledge responsible for the error. This provides a learner with feedback and allows a learner model to be updated;

3) Providing interactive support in solving problems - a technology that, without waiting for the final solution, provides a learner with intellectual assistance in solving a problem. This may be helpful to review on examples: solving new problems without highlighting errors, but using examples from previously successfully solved similar problems.

We formulate the main pedagogical and technological requirements for such systems, taking into account the above didactic requirements:

1) First of all, such a system should be integrated into the external environment, i.e. to the Internet or local network of an educational institution, to have communication tools that allow the participants in the educational process to interact and communicate with the system, to ensure their work in the network.

2) ISE should be open, which implies the possibility of using information resources in its operation, which are stored not only on local servers, but also distributed on the Internet (Web resources). To implement the properties of openness, there are various mechanisms for adequately searching for the necessary information in order to exchange and include it in the ISE database and knowledge base. These mechanisms use metadata, i.e. data about data.

3) The construction of ISE should follow the path of integration of two types of technologies adaptive and intelligent technologies, containing techniques and methods associated with various methods of implementation and functionality of ISE;

4) Intellectual technology in ISE should provide the construction of a sequence of a course of study (a 
specific sequence of learning effects) and interactive support in solving problems. Building a training course sequence means providing a learner with an individually planned sequence of classes and learning tasks. The content of educational influences is determined by the degree of concretization of the task, depending on the level of knowledge and skills of a student and this psychological portrait. It is possible to use a system of complex technology for constructing a course of study as a combination of active (with the presence of personal learning goals) and a passive (corrective) construction of a course sequence that forms it on the basis of the didactically complete material of the knowledge base of the subject area available to a student.

5) To perform the function of intelligent analysis of student responses, ISE must have a solution analyzer being implemented that allows the student to evaluate students' response and to identify students' skills and abilities based on their reasoning process modeling.

\section{Conclusion}

Thus, the article substantiates the relevance of creating a universal system capable of giving a full expert assessment of the educational activity of a specific student, and at the same time supporting the process of choosing an optimal educational path taking into account didactic rules. The system adaptability is achieved with the help of the developed "student model" being created in ISE, which corresponds to individual instructions and guidelines for various types of learning tasks. Such a model of a student can be implemented, for example, with the use of a semantic network being organized on the basis of the initial construction of an a priori model of a student based on his self-assessment. Further, in accordance with the results of the ongoing monitoring of the learning process, such a model can be redefined taking into account changes in the knowledge and experience of a learner. The system should ensure the adaptability of the presentation of course materials, navigation and testing, the division of material into didactic units. Therefore, it is necessary to provide for the possibility of a knowledge engineer of the whole course being divided into sections consisting of a set of topics. Then the corresponding models of the subject areas can be built for each of the sections of the course, which would allow more adequately to build an initial student model based on his psychological and pedagogical characteristics and test results. Of the many training influences created by a knowledge engineer in the mode of work with a student, a learning strategy is formed. When creating training scenarios, the application software packages, graphic and design, the chapters of the hypertext textbook and consultations with the system, etc. should be used (as teaching effects of problem areas).

Thus, although there are many adaptive systems for organizing support for the learning process, none of them provide the powerful functionality necessary for modern ISE that would be able to implement the full range of functions that provide: expert assessment of the quality of the knowledge provided and generated, algorithms and the functional, taking into account individual characteristics of the students, the didactic completeness of the models of subject areas, as well as the construction of developed models: students, problem areas, a teacher of applied ontology courses and adaptive models of learning.

\section{Acknowledgments}

The article is made in the framework of the program of initiative research on the topic "Intellectualization of information systems and technological processes in the field of education" (Project No. 2.9402.2017/БЧ ).

\section{References}

[1] Rybina, G. V. (2017). Intellectual technology for constructing training integrated expert systems: new opportunities. Open Education, 21(4), pp. 43-57.

[2] Gribova, V. V., Kleschev, A. S., Moskalenko, F. M., et al., (2018). The IACPaaS cloud platform for developing shells of intelligent services: state and development prospects. Software Products and Systems, 31(3), pp. 527-536.

[3] Gribova, V. V., Petryaeva, M. V., \& Fedorishchev, L. A. (2013). Computer training simulator with virtual reality for ophthalmology. Open Education, 6, pp. 45-51.

[4] Konkova, D. S., Matveev, V. A., Komissarova, O. R., et al. (2015). Analysis of the use of expert 
systems in education. Modern Scientific Research and Innovation, 12. Retrieved from http://web.snauka.ru/issues/2015/12/60271.

[5] Vagramenko, Y. A., \& Yalamov, G. Y. (2016). Analysis of the directions of intellectualization of modern information systems for educational purposes. Management of Education: Theory andPpractice, 4(24), pp. 44-56.

[6] Shikhnabiyeva, T. Sh., \& Yalamov, G. Y. (2017). On the problems of intellectualization of information systems for educational purposes in institutions of secondary vocational education and ways to solve them. Management of Education: Theory and Practice, 4, pp. 25-39.

[7] Silkina, N. S., \& Sokolinsky, L. B. (2016). Overview of adaptive models of e-learning. Bulletin of SUSU. Series: Computational Mathematics and Computer Science, 5(4), pp. 61-76.

[8] Churakova, R. G. (2001). Didactic system of L.V. Zankov. Problems and Prospects. Moscow, Russia: The Center for Developing Education. 